\title{
Shaping Ethylene Response: The Role of EIN3/EIL1 Transcription Factors
}

\author{
Vladislav A. Dolgikh ${ }^{1,2}$, Evgeniya M. Pukhovaya ${ }^{1,2}$ and Elena V. Zemlyanskaya ${ }^{1,2 *}$ \\ ${ }^{1}$ Institute of Cytology and Genetics, Siberian Branch of Russian Academy of Sciences, Novosibirsk, Russia, ${ }^{2}$ Department of \\ Natural Sciences, Novosibirsk State University, Novosibirsk, Russia
}

OPEN ACCESS

Edited by:

Jin-Song Zhang,

Institute of Genetics and

Developmental Biology (CAS),

China

Reviewed by:

Xing Wen,

Southern University of

Science and Technology,

China

Hong Qiao,

University of Texas at Austin

United States

Shangwei Zhong,

Peking University,

China

*Correspondence:

Elena V. Zemlyanskaya

ezemlyanskaya@bionet.nsc.ru

Specialty section: This article was submitted to

Plant Physiology,

a section of the journal

Frontiers in Plant Science

Received: 30 April 2019

Accepted: 23 July 2019

Published: 26 August 2019

Citation:

Dolgikh VA, Pukhovaya EM and

Zemlyanskaya EV (2019)

Shaping Ethylene Response:

The Role of EIN3/EIL1

Transcription Factors.

Front. Plant Sci. 10:1030.

doi: 10.3389/fp/s.2019.01030
EIN3/EIL1 transcription factors are the key regulators of ethylene signaling that sustain a variety of plant responses to ethylene. Since ethylene regulates multiple aspects of plant development and stress responses, its signaling outcome needs proper modulation depending on the spatiotemporal and environmental conditions. In this review, we summarize recent advances on the molecular mechanisms that underlie EIN3/EIL1directed ethylene signaling in Arabidopsis. We focus on the role of EIN3/EIL1 in tuning transcriptional regulation of ethylene response in time and space. Besides, we consider the role of EIN3/EIL1-independent regulation of ethylene signaling.

Keywords: ETHYLENE-INSENSITIVE3, ETHYLENE-INSENSITIVE3-LIKE, epigenetic regulation, protein-protein interactions, cross-talk

\section{KEY COMPONENTS OF ETHYLENE SIGNALING PATHWAY}

Plant hormone ethylene coordinates numerous developmental processes (including germination, soil emergence, seedling growth, fruit ripening, senescence, abscission, etc.), as well as diverse biotic and abiotic stress responses (Abeles et al., 2012). Ethylene has also been shown to induce typical morphological changes in dark-grown seedlings (inhibition of hypocotyl and root elongation, radial swelling of hypocotyl, and exaggeration of apical hook) known as "the triple response" (Ecker, 1995). Ethylene is produced from $L$-methionine, which is consequently converted to $S$-adenosyl$L$-methionine (by SAM-synthetases), 1-aminocyclopropane-1-carboxylic acid (ACC) (by ACC synthases), and ethylene (by ACC oxidases) (reviewed in Booker and DeLong, 2015). Ethylene is perceived by a family of receptors (ETHYLENE RESPONSE 1, ETR1; ETHYLENE RESPONSE SENSOR 1, ERS1; ETR2, ETHYLENE INSENSITIVE 4, EIN4; and ERS2 in Arabidopsis) localized in the endoplasmic reticulum (ER) membrane (reviewed in Lacey and Binder, 2014). Upon binding, ethylene inactivates them and thereby blocks the serine-threonine protein kinase CONSTITUTIVE TRIPLE RESPONSE 1 (CTR1) activity promoting the cleavage of ER-anchored EIN2 protein (reviewed in Chang, 2016; Hu et al., 2017). EIN2 C-terminal domain (EIN2-C) released upon cleavage indirectly triggers EIN3 and EIN3-Like (EIL) transcription factors (TFs) that are considered the key transcriptional regulators of ethylene response (Figure 1). Noteworthy, these TFs function as a hub that integrates and processes different cues to "shape" ethylene response in accordance with spatiotemporal and environmental conditions. Below, we will focus on the nuclear events that conduct EIN3/EIL activation and set their functional output. 


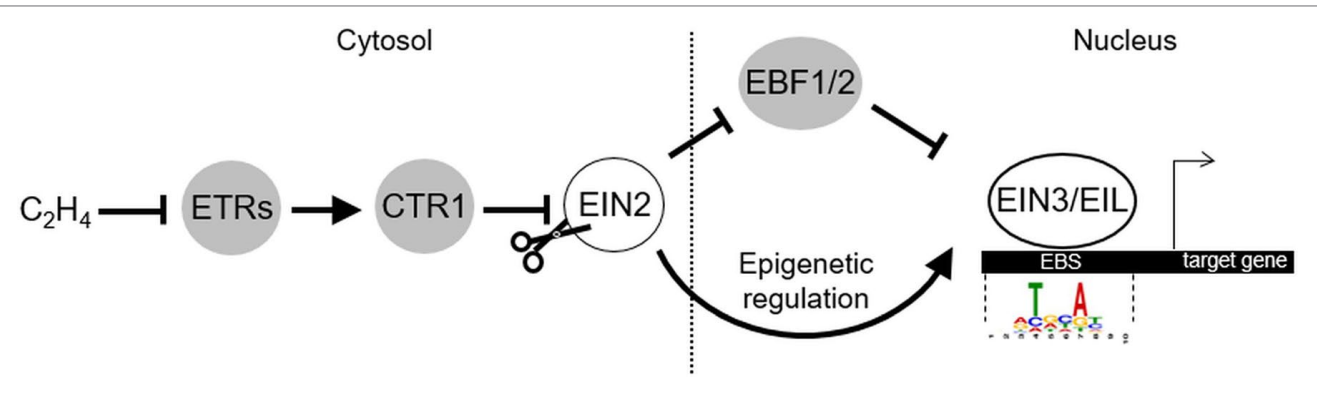

FIGURE 1 | The key components of ethylene signaling pathway. Gray and white circles depict negative and positive regulators of ethylene signaling, correspondingly. Position frequency matrix for Arabidopsis EIN3 binding motif (Chang et al., 2013) was retrieved from CIS-BP database (Weirauch et al., 2014) and visualized using Tomtom tool (http://meme-suite.org/tools/tomtom; Gupta et al. 2007). The model is based on the findings reported previously (Chang, 2016; Hu et al., 2017). The explanations are in the text. EBS, EIN3 binding site.

\section{ACTIVATION OF EIN3 AND ITS HOMOLOGS IN RESPONSE TO ETHYLENE}

EIL is a small family of plant-specific proteins. There are six genes encoding the members of this family in Arabidopsis thaliana genome (EIN3, EIL1-5) (Chao et al., 1997; Guo and Ecker, 2004). They harbor a conservative N-terminal DNA-binding domain with a unique fold structure (Song et al., 2015). EIN3, EIL1, and EIL2 represent functionally homologous proteins involved in the regulation of ethylene-responsive genes (Chao et al., 1997; Solano et al., 1998; Alonso et al., 2003; An et al., 2010). The most closely related EIN3 and EIL1 are considered the major regulators since ein 3 eil1 double mutants show complete ethylene insensitivity in terms of the triple response, pathogen resistance, and the ability to fully suppress ctr1 mutation (reviewed in Guo and Ecker, 2004; Cho and Yoo, 2015). Two paralogs differentially regulate ethylene response in the seedlings (EIN3) and in adult leaves and stems (EIL1) (An et al., 2010). Yet, a minor, EIL2 role in the regulation of ethylene response is supported by its capability to complement ein3 mutation when overexpressed (Chao et al., 1997). In Figure 2, we visualized tissue-specific expression levels of EIL genes based on publicly available data on transcriptome profiling in different Arabidopsis tissues retrieved from ThaleMine v1.10.4 (https://apps.araport.org/thalemine/; Krishnakumar et al., 2017). Unlike EIN3 and EIL1, EIL2 transcripts level is low throughout plant tissues; moderate EIL2 expression is restricted to root apical meristem and pollen (Figure 2). Therefore, EIL2 function could be limited to specific spatiotemporal conditions. EIL3/SLIM1 does not function in ethylene pathway but regulates sulfur deficiency response; no defined roles of EIL4 and EIL5 have been reported to date (reviewed in Guo and Ecker, 2004; Wawrzyńska and Sirko, 2014).
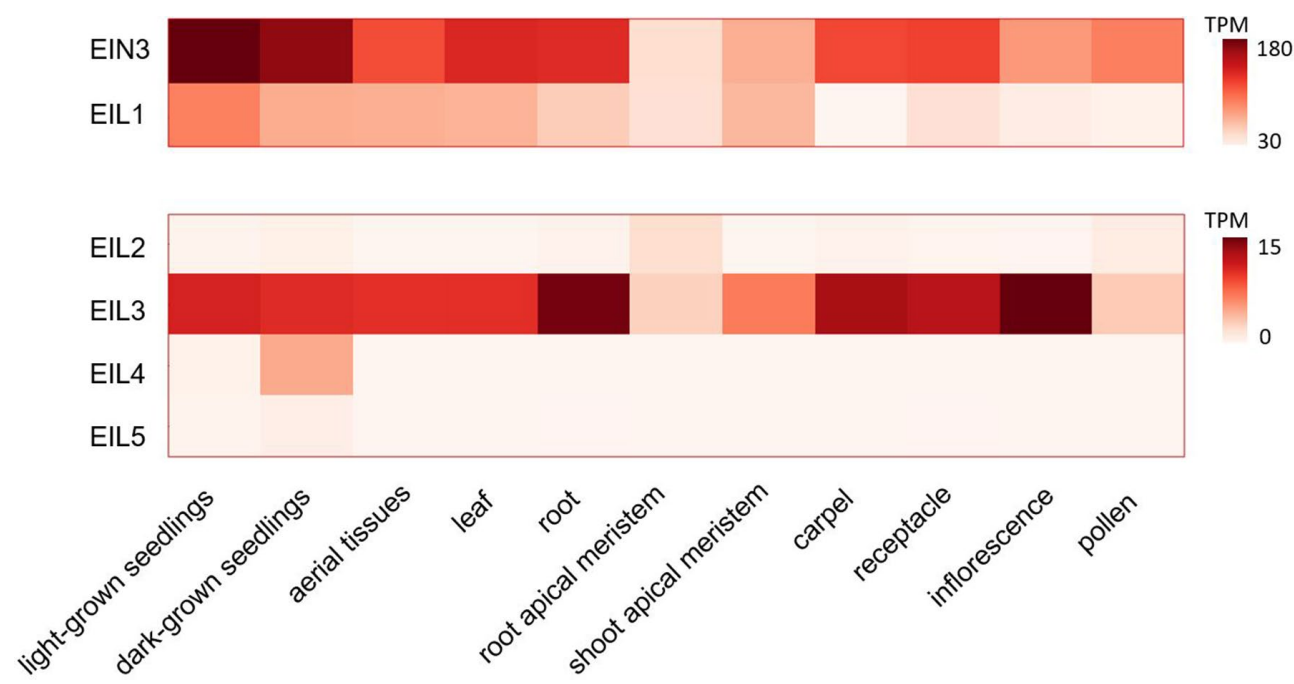

FIGURE 2 | Tissue specificity of ElL genes expression. Publicly available datasets on transcriptome profiling of different Arabidopsis tissues (light- and dark-grown seedlings (Rühl et al., 2012; Oh et al., 2014), aerial tissues (Sani et al., 2013), leaf (Wollmann et al., 2012), root (Li et al., 2013), root and shoot apical meristems (Kang et al., 2014; Nozue et al., 2015), carpel (Martínez-Fernández et al., 2014), receptacle (Niederhuth et al., 2013), inflorescence (Gan et al., 2011), pollen (Loraine et al., 2013) were used for visualization. The corresponding expression levels were retrieved from ThaleMine v1.10.4 (https://apps.araport.org/thalemine/; Krishnakumar et al., 2017). TPM, transcripts per million. 
EIN3 and EIL1 activation in response to ethylene is the target for complex regulation. EIN3 and EIL1 are short-living proteins that undergo ubiquitination and proteasomal degradation driven by ubiquitin-ligases EIN3 BINDING F-BOX1 (EBF1) and EBF2 (Figures 1 and 3A) (Gagne et al., 2004; An et al., 2010). Stabilization of EIN3/EIL1 upon ethylene release plays a pivotal role in triggering ethylene-directed gene expression. Ethylene dampens EBF1/2 levels via i) translational repression of EBF1/2 mRNA in the cytosol promoted by EIN2-C (Li et al., 2015; Merchante et al., 2015), and ii) EIN2-dependent proteasomal degradation of EBF1/2 proteins (An et al., 2010) (Figure 1). Stabilized EIN3/EIL1 accumulate in the nucleus.

EIN3/EIL1 are predominantly transcriptional activators (Chang et al., 2013; reviewed in Cho and Yoo, 2015). In Arabidopsis, EIN3, EIL1, and EIL2 specifically bind a short DNA sequence referred to as EIN3 binding site (EBS) in gene promoters (Figure 1) (Solano et al., 1998; Chang et al., 2013;
Song et al., 2015; O’Malley et al., 2016). EIN3 binds its target loci as a homodimer, and the dimerization is DNA independent (Solano et al., 1998; Song et al., 2015). Accordingly, EIN3 demonstrates higher binding affinity to the inverted repeats of EBS compared to the monomeric site in the in vitro experiments (Song et al., 2015). EIN3 binding to the targets is facilitated by elevated levels of H3K14 and non-canonical H3K23 histone acetylation both promoted by a EIN2-C-scaffolded histone acetylation complex, which is triggered upon EIN2-C interaction with a histone binding protein EIN2 NUCLEAR ASSOCIATED PROTEIN 1 (ENAP1) (Zhang et al., 2016; Zhang et al., 2017; reviewed in Wang and Qiao, 2019) (Figure 3). Since neither EIN2-C nor ENAP1 possess histone acetyltransferase domains, they might recruit other proteins to promote histone modifications. EIN3 is capable of interacting with ENAP1, too, and it is thought to contribute to ethylene-induced elevation of H3K14 and H3K23 acetylation as well (Zhang et al., 2016).

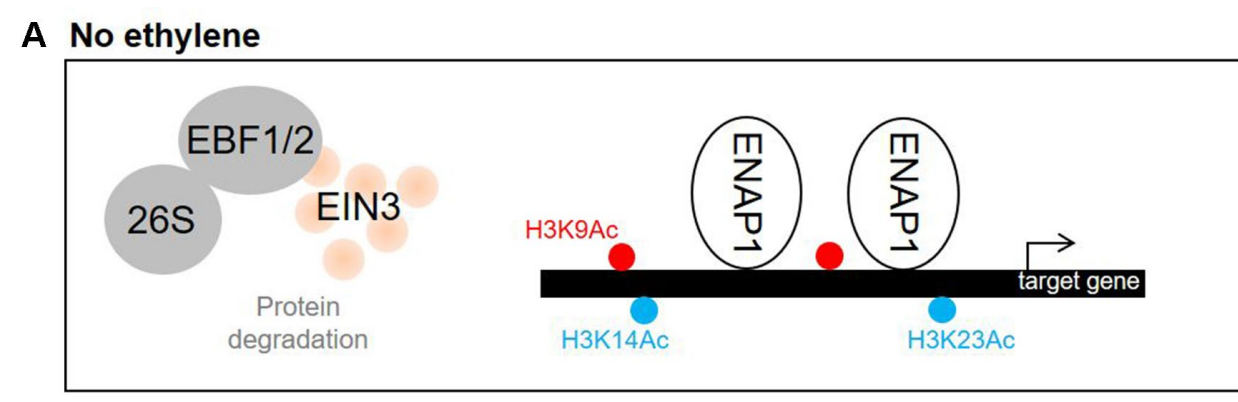

\section{B Ethylene}



FIGURE 3 | Nuclear events that promote ethylene response. (A) Without ethylene, EIN3 undergoes EBF1/2-driven degradation. (B) Upon ethylene treatment, EIN3 is stabilized. On one hand, EIN2 C-terminal domain interacts with ENAP1, which results in elevation of H3K14Ac and H3K23Ac levels, facilitated EIN3 binding to the target promoters and activation of gene expression. On the other hand, SRT1 and SRT2 histone deacetylases mediate ethylene-directed transcriptional repression by downregulating the levels of H3K9 acetylation. The models are based on the findings reported previously (Gagne et al., 2004; Li et al., 2015; Merchante et al., 2015; Zhang et al., 2016; Zhang et al., 2017; Zhang et al., 2018a). Gray and white solid circles depict negative and positive regulators of ethylene signaling, correspondingly. EIN3 is depicted in orange, H3K9Ac - in red, H3K14Ac and H3K23Ac - in blue. Dashed circles denote putative regulators (with a question mark inside) and putative regulations (with a question mark outside). HAT, histone acetyltransferase; 26S, 26S proteasome. 
Along with well-known EIN3/EIL1-promoted gene transcriptional activation, ethylene downregulates a considerable set of genes (Chang et al., 2013; Harkey et al., 2018). In a recent work, Zhang et al. (2018a) demonstrated that histone deacetylases SRT1 and SRT2 mediate transcriptional repression in response to ethylene by downregulating the levels of H3K9 acetylation (at least for a particular set of ethylene-repressed genes) (Figure 3). Both deacetylases interact with ENAP1, and the function of SRT2 is EIN2- and EIN3/EIL1-dependent. The mechanism used to distinguish between the activator and repressor pathways as well as the role of EIN3/EIL1 in SRT1/2-mediated gene repression are still unclear and need further investigations.

\section{EIN3/EIL1-REGULATED TRANSCRIPTIONAL NETWORKS}

Upon DNA binding, EIN3/EIL1 modulate multiple transcriptional cascades. Ethylene-sensitive EIN3 target genes encoding TFs include ERF1, involved in a range of ethylene responses (Solano et al., 1998), PIF3, RSL4, ESE1, and CBF1/2/3, the regulators of de-etiolation, root hair development, salt and cold stress responses, correspondingly (Zhang et al., 2011; Shi et al., 2012; Zhong et al., 2012; Feng et al., 2017). To supplement this list, numerous TF-encoding genes comprising representatives of AP2/ERF, WRKY, NAC, and other families were retrieved from whole-genome data on EIN3 binding and ethylene-induced transcriptomes (Chang et al., 2013). Besides, EIN3 directly regulates expression of chlorophyll biosynthesis genes $P O R A / B$ (Zhong et al., 2009), the pigment-binding proteins $L H C$ essential for photosynthesis initiation (Liu et al., 2017), the immune receptor FLS2 (Boutrot et al., 2010), and the apical hook formation regulator HLS1 (Lehman et al., 1996; Shen et al., 2016). EIN3/ EIL1 affect the pathways of many hormones (Chang et al., 2013), including direct regulation of hormones biosynthesis (e.g., salicylic acid biosynthesis gene SID2, Chen et al., 2009), and signaling (e.g., type-A negative regulators of cytokinin signaling $A R R 5 / 7 / 15$, Shi et al., 2012). To maintain a homeostasis, EIN3 activates a feedback regulatory circuit by inducing transcription of EBF2 (Konishi and Yanagisawa, 2008) and probably some other negative regulators of ethylene signaling (Chang et al., 2013).

To provide a proper phenotypic outcome upon ethylene release, these transcriptional cascades and the downstream growth control pathways should be tightly coordinated, which is supported by data on the dynamic changes of ethyleneinduced transcriptomes in etiolated Arabidopsis seedlings where four distinct transcriptional waves are segregated (Chang et al., 2013). The observed transcription kinetics may be due to distinct mechanisms of transcriptional control, or the heterogeneity of the ethylene response in different tissues (Chang et al., 2013). Transcriptome profiling of Arabidopsis mutants identified large groups of EIN3/EIL1-regulated genes that were co-regulated by the other TFs such as RHD6 (root hair development) and PIFs (light signaling) (Feng et al., 2017; Shi et al., 2018), which implies co-regulation of EIN3/ EIL1-triggered transcription by certain developmental and environmental cues. In the following sections, we illustrate that EIN3/EIL1 proteins represent crucial targets for tuning the downstream transcriptional cascades in time and space.

\section{TUNING TRANSCRIPTIONAL REGULATION OF ETHYLENE RESPONSE}

\section{Epigenetic Regulation of Spatiotemporal Expression of EIN3/EIL1 Target Genes}

Climacteric fleshy fruits (the ones that demonstrate a respiratory burst at the beginning of ripening) use ethylene as a ripening signal (McMurchie et al., 1972). Mature fruit produces ethylene in an autocatalytic manner (system II) unlike immature fruit and vegetative tissues where self-inhibitory ethylene production (system I) is implemented. Autocatalytic regulation suggests a positive feedback loop controlling ethylene synthesis. Presumably, the corresponding regulatory circuit includes EIN3 triggered transcriptional cascade that finally activates ethylene biosynthesis genes (ACSes and ACOs) (Vandenbussche et al., 2012; Lü et al., 2018). To prevent uncontrolled ethylene production, this circuit should be under a tight spatiotemporal regulation.

Epigenetic modifications often promote spatiotemporal regulation of plant hormone responses (reviewed in Yamamuro et al., 2016). In Arabidopsis, a repressive mark H3K27me3 regulates expression of a large number of genes (Lafos et al., 2011). A systematic analysis of epigenome and transcriptome data suggests that climacteric fruits use removal of H3K27me3 to trigger autocatalytic system II ethylene production specifically in the mature fruit (Lü et al., 2018). Accordingly, EIN3 targeted promoters-a part of transcriptional feedback circuit controlling climacteric fruit ripening ( $R I N$ in tomato, NAC in peach and banana)-are associated with the repressive histone mark H3K27me3 in leaf and immature fruit. They become demethylated and therefore accessible only in the ripening fruit tissues. Presumably, this epigenetic mechanism prevents autocatalytic ethylene production in vegetative and immature fruit tissues.

Recently, using a systematic analysis of publicly available ChIP-Seq data on EIN3 binding in Arabidopsis, we have demonstrated that EIN3 direct targets are enriched in a chromatin state 4 according to the classification of Sequeira-Mendes et al. (2014), which is associated with $\mathrm{H} 3 \mathrm{~K} 27 \mathrm{me} 3$ repressive mark (Zemlyanskaya et al., 2017b). Therefore, H3K27me3-associated epigenetic silencing might be a more general mechanism providing spatiotemporal specificity of ethylene response via restriction of EIN3 function.

\section{Modulation of EIN3/EIL1 Protein Stability}

Regulation of EIN3/EIL1 levels via the control of the protein stability by EBF1/2 is a pivotal mechanism of EIN3/EIL1 adjustment in ethylene signaling. Simultaneously, it can be affected by environmental factors resulting in a modulation of transcriptional response to ethylene. Plants germinating in the darkness assume a light-regulated developmental program known as skotomorphogenesis, which phenotypically results in rapid hypocotyl elongation, small closed chlorotic cotyledons, and apical hook formation (McNellis and Deng, 1995). 
EIN3/EIL1 and their target genes (e.g., HLS1, ERF1, PIF3, $P O R A / B$ ) play essential roles in these processes. They contribute in chlorosis and increased apical hook curvature of buried seedlings, induce shortening and thickening of hypocotyl to enhance lifting capacity of the seedling, and finally promote seedlings greening upon light irradiation (Zhong et al., 2009; Zhong et al., 2012; Zhong et al., 2014; Shen et al., 2016).

In the seedlings growing through the soil, EIN3/EIL1 are stabilized by both light signaling and ethylene, which accumulates in response to mechanical pressure. In the former case, E3 ubiquitin ligase CONSTITUTIVE PHOTOMORPHOGENIC 1 (COP1), a central repressor of light signaling, directly targets EBF1/2 for ubiquitination and degradation (Shi et al., 2016a). As seedlings grow toward the surface, light intensity gradually increases. As a result, COP1 activity, which is negatively regulated by photoreceptors (Podolec and Ulm, 2018), gradually decreases attenuating ethylene response.

When the seedling reaches the soil surface, light triggers a dramatic developmental transition known as de-etiolation that leads to immediate termination of ethylene responses. Light-activated photoreceptor phytochrome B (phyB) directly interacts with both EIN3 and EBF1/2 proteins, thereby stimulating robust EIN3 degradation, rapidly turning off ethylene signaling (Shi et al., 2016b; Luo and Shi, 2019).

\section{Repression of EIN3/EIL1 Transcriptional Activity}

In this section, we consider the cross-talk of ethylene signaling pathway with jasmonic acid (JA) and gibberellins (GA) based on an inhibition of EIN3/EIL1 transcriptional activity due to their physical interactions with repressor proteins (Table 1). These proteinprotein interactions (PPI) rather prevent EIN3/EIL1 binding to DNA than cause changes in protein stability (Zhu et al., 2011; An et al., 2012; Zhang et al., 2014). JA and ethylene synergistically regulate certain aspects of plant development (such as root hair development and inhibition of root growth) and tolerance to necrotrophic fungi. The transcriptional repressors JASMONATE ZIM-DOMAIN (JAZ) are the master regulators that interact with MYC2, MYC3, and MYC4 TFs and negatively control JA signaling (reviewed in Wasternack and Song, 2017). JAZ proteins interact with EIN3/EIL1 and enhance EIN3/EIL1 binding to HDA6, an RPD-type histone deacetylase (Zhu et al., 2011; Zhu and Lee, 2015). The resulting complex inhibits EIN3/EIL1-mediated transcription. Upon JA treatment, JAZ degrades, attenuating HDA6-EIN3/ EIL1 association and therefore activating EIN3/EIL1. Therefore, pathogenesis-related genes ERF1 and ORA59, directly regulated by EIN3/EIL1, as well as their downstream target PDF1.2, are upregulated in response to JA.

At the same time, MYC2, MYC3, and MYC4 transcriptional regulators of JA signaling interact with EIN3/EIL1, inhibiting their function (Song et al., 2014; Zhang et al., 2014). Thus, ERF1, ORA59, and PDF1.2 genes are upregulated in myc2 mutants. This inhibitory mechanism underlies ethylene-JA antagonism. Particularly, JA-directed abolishment of ethylene-promoted apical hook formation proceeds via MYC2-mediated attenuation of HOOKLESS1 (HLS1) expression, which is the key regulator of hook development and a direct EIN3/EIL1 target (Lehman et al., 1996; An et al., 2012; Song et al., 2014; Zhang et al., 2014). Additionally, MYC2 targets $E B F 1$, inducing its expression and therefore promoting EIN3/EIL1 degradation (Zhang et al., 2014). Noteworthy, the inhibitory effect in the EIN3-MYC2 complex is reciprocal: the interaction suppresses MYC2 activity as well and thereby ethylene attenuates JA-regulated plant defense response against insect attack (Song et al., 2014). Similarly, EIN3 plays an inhibitory role in sulfur deficiency response, forming heterodimers with EIL3/SLIM1 TF and preventing its target gene recognition by EIL3/SLIM1 (Wawrzyńska and Sirko, 2016).

Just as in the case of JA-ethylene synergy, GA enhances apical hook curvature at least partially via a release of EIN3/EIL1 from repressor proteins. DELLA proteins are the main transcriptional repressors of GA responses (Sun and Gubler, 2004). Two members of this family (RGA and GAI) are capable of associating with EIN3/EIL1 DNA-binding domain and inhibiting EIN3/EIL1 function (An et al., 2012). In response to GAs, DELLA proteins rapidly degrade, thereby de-repressing EIN3/EIL1-mediated transcription of at least the HLS1 gene.

\section{EIN3/EIL1 Cooperate With Other TFs in an Interdependent Manner}

EIN3/EIL1's capability to function cooperatively with the transcriptional regulators of the other signaling pathways provides another possibility to shape spatiotemporal patterns of ethylene response. This cooperation implies the cross-talk of TFs bound to DNA that goes along with the physical interaction of these TFs (Table 1). In buried seedlings, the chloroplasts' development is arrested at the etioplast stage, characterized by an immature arrangement of the inner membranes and pigment molecules (Solymosi and Schoefs, 2010; Jarvis and López-Juez, 2013). EIN3 and PHYTOCHROME INTERACTING FACTOR3 (PIF3), a darkness-stabilized transcriptional regulator of light signaling, form an interdependent module that represses chloroplast development in buried seedlings (Liu et al., 2017). Namely, EIN3 and PIF3 directly interact and bind the promoters of LIGHT HARVESTING COMPLEX ( $L H C)$ genes in a cooperative manner to synergistically suppress their expression. Upon light exposure, the levels of EIN3 and PIF3 decrease, and activation of LHC expression triggers chloroplast differentiation.

Interestingly, another TF from PIF family, PIF4, interacts with EIN3 as well (Yazaki et al., 2016), and both TFs target HLS1, the key regulator of apical hook development (An et al., 2012; Zhang et al., 2018b). However, EIN3 and PIF4 activate HLS1 transcription independently (Zhang et al., 2018b).

Cooperative regulation also guides ethylene functioning in root hair development. EIN3 promotes root hair elongation by directly activating RHD6-LIKE4 (RSL4) gene (Feng et al., 2017). Besides, EIN3 physically interacts with ROOT HAIR DEFECTIVE6 (RHD6), a major regulator of root hair development that targets RSL4 as well (Yi et al., 2010; Feng et al., 2017). Both EIN3 and RHD6 co-activate RSL4 more efficiently than either of them alone (Feng et al., 2017). The role of EIN3-RHD6 cooperative action is most likely not limited to RSL4 regulation, but rather covers a quite extensive set of genes and contributes to ethylene-promoted 
root hair initiation as well (Feng et al., 2017). Similarly, in papaya, EIN3 homolog CpEIN3a interacts with CpNAC2, and both TFs directly activate the transcription of carotenoid biosynthesisrelated genes $C p P D S 4$ and $C p C H Y-b$ expressed during fruit ripening (Fu et al., 2017). Both TFs possess a combinatory effect on the regulation of their targets.

Besides, EIN3/EIL1 are capable of binding gene promoters and affecting gene expression indirectly via physical interactions with other TFs and modulation of their activity (Table 1). Increase of auxin biosynthesis in the root tip epidermis via upregulation of TRYPTOPHAN AMINOTRANSFERASE OF ARABIDOPSIS 1 (TAA1) plays a pivotal role in ethylene-induced inhibition of root growth (Vaseva et al., 2018). EIN3 targets TAA1 promoter through a "piggyback" interaction with RESPONSE REGULATOR 1 (ARR1), a transcriptional regulator of cytokinin signaling, thereby enhancing ARR1 transcriptional activity (Yan et al., 2017). Similarly, EIN3/EIL1 interact with FER-LIKE FE DEFICIENCYINDUCED TRANSCRIPTION FACTOR (FIT), a central regulator of $\mathrm{Fe}$ acquisition in roots, activating FIT abundance (Lingam et al., 2011). Moreover, EIN3/EIL1 bridges FIT to the transcriptional Mediator complex to recruit RNA-pol and promote the regulation of iron homeostasis (Yang et al., 2014).

\section{EIN3/EIL1-INDEPENDENT ETHYLENE SIGNALING}

There is growing evidence that despite their essential role, EIN3/EIL1 TFs are not indispensable components of ethylene response. Thus, kinetic studies distinguish two phases of ethylene-induced growth inhibition of the hypocotyl in etiolated Arabidopsis seedlings: a transient phase I (up to $2 \mathrm{~h}$ ) and a sustained phase II (Binder et al., 2004; Chang et al., 2013). Both phases require EIN2 function, while only the second requires EIN3/EIL1 (Binder et al., 2004). Intriguingly, unlike etiolated seedlings, light-grown ein3 eil1 double mutants do not demonstrate the total loss of longterm ethylene responses (Harkey et al., 2018). Moreover, osmotic stress-induced cell cycle arrest in leaf primordia that coincides with enhanced activation of the ethylene signal is EIN3 independent (Skirycz et al., 2011). These observations favor the existence of an alternative pathway. One possible candidate to promote such regulation is a PAM domaincontaining protein EER5. It negatively regulates ethylene signaling during hypocotyl elongation in etiolated seedlings regardless of EIN3 by promoting downregulation of a gene subset upon ethylene treatment. In addition, it physically interacts with EIN2-C (Christians et al., 2008). EER5 regulates magnitude of ethylene response via perception of ERS1 signal (Deslauriers et al., 2015).

\section{CONCLUDING REMARKS AND PERSPECTIVES}

Ethylene response is a target for a complex regulation, in which EIN3/EIL1 TFs play a crucial role. Recent studies shed light on multiple layers of complexity in tuning EIN3/EIL1 function (including epigenetic gene silencing and modulation of EIN3/EIL1 stability and activity via PPIs) that facilitate the

TABLE 1 | Protein-protein interactions involved in modulation of EIN3/EIL1 function.

\begin{tabular}{|c|c|c|c|c|c|c|}
\hline Protein & Organism & Pathway & Function & PPI targets & Interaction output & Reference \\
\hline \multicolumn{7}{|c|}{ EIN3/EIL1 stability } \\
\hline $\mathrm{EBF} 1 / 2$ & Arabidopsis thaliana & Ethylene signaling & F-box protein & EIN3/EIL1 & EIN3/EIL1 degradation & $\begin{array}{l}\text { Gagne et al., 2004; } \\
\text { An et al., } 2010\end{array}$ \\
\hline COP1 & Arabidopsis thaliana & Light signaling & E3 ubiquitin ligase & EBF1/2 & EIN3/EIL1 stabilization & Shi et al., 2016a \\
\hline phyB & Arabidopsis thaliana & Light signaling & Protein binding & EIN3/EIL1, EBF1/2 & EIN3 degradation & Shi et al., 2016b \\
\hline AKIN10 & Arabidopsis thaliana & Catabolic pathways & PK & EIN3 & EIN3 degradation & Kim et al., 2017 \\
\hline \multicolumn{7}{|c|}{ EIN3/EIL1 repression } \\
\hline RGA, GAI & Arabidopsis thaliana & GA signaling & $\mathrm{RP}$ & EIN3/EIL1/2 & EIN3/EIL1 repression & An et al., 2012 \\
\hline JAZ1 & Arabidopsis thaliana & JA signaling & $\mathrm{RP}$ & EIN3/EIL1, HDA6 & $\begin{array}{l}\text { EIN3/EIL1 repression in } \\
\text { complex with HDA6 }\end{array}$ & Zhu et al., 2011 \\
\hline $\mathrm{MYC} 2 / 3 / 4$ & Arabidopsis thaliana & JA signaling & $\mathrm{TF}$ & EIN3/EIL1 & EIN3/EIL1 repression & $\begin{array}{l}\text { Song et al., 2014; } \\
\text { Zhang et al., } 2014\end{array}$ \\
\hline EIL3/SLIM & Arabidopsis thaliana & $\begin{array}{l}\text { Sulfur deficiency } \\
\text { response }\end{array}$ & $\mathrm{TF}$ & EIN3 & EIL3/SLIM repression & $\begin{array}{l}\text { Wawrzyńska and } \\
\text { Sirko, } 2016\end{array}$ \\
\hline \multicolumn{7}{|c|}{ EIN3/EIL1 cooperation with other TFs } \\
\hline RHD6 & Arabidopsis thaliana & Root hair formation & TF & EIN3 & RSL4 co-activation & Feng et al., 2017 \\
\hline PIF3 & Arabidopsis thaliana & Light signaling & & EIN3 & LHC co-repression & Liu et al., 2017 \\
\hline CpNAC2 & Carica papaya L. & Carotenoid biosynthe & isTF & CpEIN3a & $\begin{array}{l}\text { CpPDS4 and } \mathrm{CpCHY-b} \\
\text { co-activation }\end{array}$ & Fu et al., 2017 \\
\hline FIT & Arabidopsis thaliana & $\begin{array}{l}\text { Iron acquisition } \\
\text { pathway }\end{array}$ & $\mathrm{TF}$ & EIN3/EIL1 & FIT stabilization & Lingam et al., 2011 \\
\hline MED25 & Arabidopsis thaliana & $\mathrm{N} / \mathrm{A}$ & Mediator subunit & EIN3/EIL1 & FIT activation & Yang et al., 2014 \\
\hline ARR1 & Arabidopsis thaliana & Cytokinin signaling & TF & EIN3 & ARR1 activation & Yan et al., 2017 \\
\hline
\end{tabular}

PPI, protein-protein interaction; JA, jasmonic acid; GA, gibberellins; TF, transcription factor; RP, repressor protein; PK, protein kinase. 
"shaping" of ethylene response according to spatiotemporal and environmental conditions. At the same time, these findings open up new perspectives for further research. Growing evidence of the important role that epigenetic landscape plays in EIN3/ EIL1 functioning requires its more detailed characterization. Particularly, the contribution of distinct epigenetic modifications as well as ENAP1 patterns in modulation of EIN3/EIL1 function is of interest. In view of interdependent cooperation of EIN3/ EIL1 with some TFs described recently, the detailed analysis of nucleotide context surrounding EIN3 binding sites requires more attention, and genome-wide research appears helpful both to generalize resent findings and to predict new connections. Moreover, it would be interesting to clarify the role of epigenetic regulation and PPIs in suppression of gene expression upon ethylene treatment.

Yet, despite their essential role, EIN3/EIL1 are not indispensable regulators of ethylene response. To couple the molecular events and phenotypic responses more precisely, EIL2 function in ethylene signaling and EIN3/EIL independent pathways are to be elucidated.

\section{REFERENCES}

Abeles, F. B., Morgan, P. W., and Saltveit, M. E., Jr. (2012). Ethylene in plant biology. Academic press. San Diego, CA.

Alonso, J. M., Stepanova, A. N., Solano, R., Wisman, E., Ferrari, S., Ausubel, F. M., et al. (2003). Five components of the ethylene-response pathway identified in a screen for weak ethylene-insensitive mutants in Arabidopsis. Proc. Natl. Acad. Sci. U.S.A. 100, 2992-1997. doi: 10.1073/pnas.0438070100

An, F., Zhao, Q., Ji, Y., Li, W., Jiang, Z., Yu, X., et al. (2010). Ethylene-induced stabilization of ETHYLENE INSENSITIVE3 and EIN3-LIKE1 is mediated by proteasomal degradation of EIN3 binding F-box 1 and 2 that requires EIN2 in Arabidopsis. Plant Cell 22, 2384-2401. doi: 10.1105/tpc.110.076588

An, F., Zhang, X., Zhu, Z., Ji, Y., He, W., Jiang, Z., et al. (2012). Coordinated regulation of apical hook development by gibberellins and ethylene in etiolated Arabidopsis seedlings. Cell Res. 22, 915-927. doi: 10.1038/cr.2012.29

Binder, B. M., Mortimore, L. A., Stepanova, A. N., Ecker, J. R., and Bleecker, A. B. (2004). Short-term growth responses to ethylene in Arabidopsis seedlings are EIN3/EIL1 independent. Plant Physiol. 136, 2921-2927. doi: 10.1104/ pp.104.050393

Booker, M. A., and DeLong, A. (2015). Producing the ethylene signal: regulation and diversification of ethylene biosynthetic enzymes. Plant Physiol. 169, 42-50. doi: $10.1104 /$ pp.15.00672

Boutrot, F., Segonzac, C., Chang, K. N., Qiao, H., Ecker, J. R., Zipfel, C., et al. (2010). Direct transcriptional control of the Arabidopsis immune receptor FLS2 by the ethylene-dependent transcription factors EIN3 and EIL1. Proc. Natl. Acad. Sci. U.S.A. 107, 14502-14507. doi: 10.1073/pnas.1003347107

Chang, C. (2016). Q\&A: how do plants respond to ethylene and what is its importance? BMC Biol. 14, 7. doi: 10.1186/s12915-016-0230-0

Chang, K. N., Zhong, S., Weirauch, M. T., Hon, G., Pelizzola, M., Li, H., et al. (2013). Temporal transcriptional response to ethylene gas drives growth hormone cross-regulation in Arabidopsis. Elife 2, e00675. doi: 10.7554/eLife.00675

Chao, Q., Rothenberg, M., Solano, R., Roman, G., Terzaghi, W., and Ecker, J. R. (1997). Activation of the ethylene gas response pathway in Arabidopsis by the nuclear protein ETHYLENE-INSENSITIVE3 and related proteins. Cell 89, 1133-1144. doi: 10.1016/S0092-8674(00)80300-1

Chen, H., Xue, L., Chintamanani, S., Germain, H., Lin, H., Cui, H. et al. (2009). ETHYLENE INSENSITIVE3 and ETHYLENE INSENSITIVE3-LIKE1 repress SALICYLIC ACID INDUCTION DEFICIENT2 expression to negatively regulate plant innate immunity in Arabidopsis. Plant Cell 21, 2527-2540. doi: $10.1105 /$ tpc. 108.065193

\section{AUTHOR CONTRIBUTIONS}

VAD and EMP performed the literature search and drafted the paper. VAD performed the analysis of the transcriptome datasets. EVZ revised and edited the manuscript. All authors read and approved the final manuscript.

\section{FUNDING}

The work was funded by the Russian Foundation for Basic Research through grant № 18-29-13040. Meta-analysis of the transcriptome datasets was done in the frame of the project supported by the Russian Foundation for Basic Research and the government of Novosibirsk region through grant № 18-44-540039.

\section{ACKNOWLEDGMENTS}

We thank Nadya Omelyanchuk for the critical reading of the manuscript and for fruitful discussions.

Cho, Y. H., and Yoo, S. D. (2015). Novel connections and gaps in ethylene signaling from the ER membrane to the nucleus. Front. Plant Sci. 5, 733. doi: 10.3389/ fpls.2014.00733

Christians, M. J., Robles, L. M., Zeller, S. M., and Larsen, P. B. (2008). The eer5 mutation, which affects a novel proteasome-related subunit, indicates a prominent role for the COP9 signalosome in resetting the ethylene-signaling pathway in Arabidopsis. Plant J. 55, 467-577. doi: 10.1111/j.1365-313X.2008.03521.x

Deslauriers, S. D., Alvarez, A. A., Lacey, R. F., Binder, B. M., and Larsen, P. B. (2015). Dominant gain-of-function mutations in transmembrane domain III of ERS1 and ETR1 suggest a novel role for this domain in regulating the magnitude of ethylene response in Arabidopsis. New Phytol. 208, 442-455. doi: 10.1111/nph.13466

Ecker, J. R. (1995). The ethylene signal transduction pathway in plants. Science 268, 667-675. doi: 10.1126/science.7732375

Feng, Y., Xu, P., Li, B., Li, P., Wen, X., An, F., et al. (2017). Ethylene promotes root hair growth through coordinated EIN3/EIL1 and RHD6/RSL1 activity in Arabidopsis. Proc. Natl. Acad. Sci. U.S.A. 114, 13834-13839. doi: 10.1073/pnas.1711723115

Fu, C. C., Han, Y. C., Kuang, J. F., Chen, J. Y., and Lu, W. J. (2017). Papaya CpEIN3a and CpNAC2 co-operatively regulate carotenoid biosynthesis-related genes CpPDS2/4, CpLCY-e and CpCHY-b during fruit ripening. Plant Cell Physiol. 58, 2155-2165. doi: 10.1093/pcp/pcx149

Gagne, J. M., Smalle, J., Gingerich, D. J., Walker, J. M., Yoo, S., Yanagisawa, S., et al. (2004). Ubiquitin-protein ligases that repress ethylene action and promote growth by directing EIN3 degradation. Proc. Natl. Acad. Sci. 101, 6803-6808. doi: 10.1073/pnas.0401698101

Gan, X., Stegle, O., Behr, J., Steffen, J. G., Drewe, P., and Hildebrand, K. L. (2011). Multiple reference genomes and transcriptomes for Arabidopsis thaliana. Nature 477, 419-423. doi: 10.1038/nature10414

Guo, H., and Ecker, J. R. (2004). The ethylene signaling pathway: new insights. Curr. Opin. Plant Biol. 7, 40-49. doi: 10.1016/j.pbi.2003.11.011

Gupta, S., Stamatoyannopoulos, J. A., Bailey, T. L., and Noble, W. S. (2007). Quantifying similarity between motifs. Genome Biol. 8, R24. doi: 10.1186/ gb-2007-8-2-r24

Harkey, A. F., Watkins, J. M., Olex, A. L., DiNapoli, K. T., Lewis, D. R., Fetrow, J. S., et al. (2018). Identification of transcriptional and receptor networks that control root responses to ethylene. Plant Physiol. 176, 2095-2118. doi: 10.1104/pp.17.00907

$\mathrm{Hu}, \mathrm{Y}$, Vandenbussche, F., and Van Der Straeten, D. (2017). Regulation of seedling growth by ethylene and the ethylene-auxin crosstalk. Planta 245, 467-489. doi: 10.1007/s00425-017-2651-6 
Jarvis, P., and López-Juez, E. (2013). Biogenesis and homeostasis of chloroplasts and other plastids. Nat. Rev. Mol. Cell. Biol. 14, 787-802. doi: 10.1038/nrm3702

Kang, J., Yu, H., Tian, C., Zhou, W., Li, C., Jiao, Y., et al. (2014). Suppression of photosynthetic gene expression in roots is required for sustained root growth under phosphate deficiency. Plant Physiol. 165, 1156-1170. doi: 10.1104/ pp.114.238725

Kim, G. D., Cho, Y. H., and Yoo, S. D. (2017). Regulatory functions of cellular energy sensor SNF1-Related Kinase1 for leaf senescence delay through ETHYLENEINSENSITIVE3 repression. Sci. Rep. 7, 3193. doi: 10.1038/s41598-017-03506-1

Konishi, M., and Yanagisawa, S. (2008). Ethylene signaling in Arabidopsis involves feedback regulation via the elaborate control of EBF2 expression by EIN3. Plant J. 55, 821-831. doi: 10.1111/j.1365-313X.2008.03551.x

Krishnakumar, V., Contrino, S., Cheng, C. Y., Belyaeva, I., Ferlanti, E. S., Miller, J. R., et al. (2017). ThaleMine: a warehouse for Arabidopsis data integration and discovery. Plant Cell Physiol. 58, e4. doi: 10.1093/pcp/pcw200

Lacey, R. F., and Binder, B. M. (2014). How plants sense ethylene gas-The ethylene receptors. J. Inorg. Biochem. 133, 58-62. doi: 10.1016/j.jinorgbio.2014.01.006

Lafos, M., Kroll, P., Hohenstatt, M.L., Thorpe, F.L., Clarenz, O., and Schubert, D. (2011). Dynamic regulation of H3K27 trimethylation during Arabidopsis differentiation. PLoS Genet. 7, e1002040. doi: 10.1371/journal.pgen.1002040

Lehman, A., Black, R., and Ecker, J. R. (1996). HOOKLESS1, an ethylene response gene, is required for differential cell elongation in the Arabidopsis hypocotyl. Cell 85, 183-194. doi: 10.1016/S0092-8674(00)81095-8

Li, W., Lin, W. D., Ray, P., Lan, P., and Schmidt, W. (2013). Genome-wide detection of condition-sensitive alternative splicing in Arabidopsis roots. Plant Physiol. 162, 1750-1763. doi: 10.1104/pp.113.217778

Li, W., Ma, M., Feng, Y., Li, H., Wang, Y., Ma, Y., et al. (2015). EIN2-directed translational regulation of ethylene signaling in Arabidopsis. Cell 163, 670-683. doi: 10.1016/j.cell.2015.09.037

Lingam, S., Mohrbacher, J., Brumbarova, T., Potuschak, T., Fink-Straube, C., Blondet, E., et al. (2011). Interaction between the bHLH transcription factor FIT and ETHYLENE INSENSITIVE3/ETHYLENE INSENSITIVE3-LIKE1 reveals molecular linkage between the regulation of iron acquisition and ethylene signaling in Arabidopsis. Plant Cell. 23, 1815-1829. doi: 10.1105/ tpc.111.084715

Liu, X., Liu, R., Li, Y., Shen, X., Zhong, S., and Shi, H. (2017). EIN3 and PIF3 form an interdependent module that represses chloroplast development in buried seedlings. Plant Cell 29, 3051-3067. doi: 10.1105/tpc.17.00508

Loraine, A. E., McCormick, S., Estrada, A., Patel, K., and Qin, P. (2013). RNA-seq of Arabidopsis pollen uncovers novel transcription and alternative splicing. Plant Physiol. 162, 1092-1109. doi: 10.1104/pp.112.211441

Lü, P., Yu, S., Zhu, N., Chen, Y. R., Zhou, B., Pan, Y., et al. (2018). Genome encode analyses reveal the basis of convergent evolution of fleshy fruit ripening. Nat. Plants 4, 784-791. doi: 10.1038/s41477-018-0249-z

Luo, Y., and Shi, H. (2019). Direct regulation of phytohormone actions by photoreceptors. Trends Plant Sci. 24, 105-108. doi: 10.1016/j.tplants.2018.11.002

Martínez-Fernández, I., Sanchís, S., Marini, N., Balanzá, V., Ballester, P., Navarrete-Gómez, M., et al. (2014). The effect of NGATHA altered activity on auxin signaling pathways within the Arabidopsis gynoecium. Front. Plant Sci. 5, 210. doi: $10.3389 /$ fpls.2014.00210

Merchante, C., Brumos, J., Yun, J., Hu, Q., Spencer, K. R., Enríquez, P., et al. (2015). Gene-specific translation regulation mediated by the hormone-signaling molecule EIN2. Cell 163, 684-697. doi: 10.1016/j.cell.2015.09.036

McMurchie, E. J., McGlasson, W. B., and Eaks., I. L. (1972). Treatment of fruit with propylene gives information about the biogenesis of ethylene. Nature 237, 235-236. doi: 10.1038/237235a0

McNellis, T. W., and Deng, X. W. (1995). Light control of seedling morphogenetic pattern. Plant Cell 7, 1749-1761. doi: 10.1105/tpc.7.11.1749

Niederhuth, C. E., Patharkar, O. R., and Walker, J. C. (2013). Transcriptional profiling of the Arabidopsis abscission mutant hae hsl2 by RNA-Seq. BMC Genomics 14, 37. doi: 10.1186/1471-2164-14-37

Nozue, K., Tat, A. V., Kumar Devisetty, U., Robinson, M., Mumbach, M. R., Ichihashi, Y., et al. (2015). Shade avoidance components and pathways in adult plants revealed by phenotypic profiling. PLoS Genet. 11, e1004953. doi: 10.1371/journal.pgen.1004953

Oh, E., Zhu, J. Y., Bai, M. Y., Arenhart, R. A., Sun, Y., and Wang, Z. Y. (2014). Cell elongation is regulated through a central circuit of interacting transcription factors in the Arabidopsis hypocotyl. Elife 3, e03031. doi: 10.7554/eLife.03031
O’Malley, R. C., Huang, S. C., Song, L., Lewsey, M. G., Bartlett, A., Nery, J. R., et al. (2016). Cistrome and epicistrome features shape the regulatory DNA landscape. Cell 165, 1280-1292. doi: 10.1016/j.cell.2016.04.038

Podolec, R., and Ulm, R. (2018). Photoreceptor-mediated regulation of the COP1/ SPA E3 ubiquitin ligase. Curr. Opin. Plant Biol. 45 (Pt A), 18-25. doi: 10.1016/ j.pbi.2018.04.018

Rühl, C., Stauffer, E., Kahles, A., Wagner, G., Drechsel, G., Rätsch, G., et al. (2012). Polypyrimidine tract binding protein homologs from Arabidopsis are key regulators of alternative splicing with implications in fundamental developmental processes. Plant Cell 24, 4360-4375. doi: 10.1105/tpc.112.103622

Sani, E., Herzyk, P., Perrella, G., Colot, V., and Amtmann, A. (2013). Hyperosmotic priming of Arabidopsis seedlings establishes a long-term somatic memory accompanied by specific changes of the epigenome. Genome Biol. 14, R59. doi: 10.1186/gb-2013-14-6-r59

Shen, X., Li, Y., Pan, Y., and Zhong, S. (2016). Activation of HLS1 by mechanical stress via ethylene-stabilized EIN3 is crucial for seedling soil emergence. Front. Plant. Sci. 7, 1571. doi: 10.3389/fpls.2016.01571

Shi, Y., Tian, S., Hou, L., Huang, X., Zhang, X., Guo, H., et al. (2012). Ethylene signaling negatively regulates freezing tolerance by repressing expression of CBF and type-A ARR genes in Arabidopsis. Plant Cell. 24, 2578-2595. doi: 10.1105/tpc.112.098640

Shi, H., Lyu, M., Luo, Y., Liu, S., Li, Y., He, H., et al. (2018). Genome-wide regulation of light-controlled seedling morphogenesis by three families of transcription factors. Proc. Natl. Acad. Sci. U.S.A. 115, 6482-6487. doi: 10.1073/ pnas. 1803861115

Shi, H., Liu, R., Xue, C., Shen, X., Wei, N., Deng, X. W., et al. (2016a). Seedlings transduce the depth and mechanical pressure of covering soil using COP1 and ethylene to regulate EBF1/EBF2 for soil emergence. Curr. Biol. 26, 139-149. doi: 10.1016/j.cub.2015.11.053

Shi, H., Shen, X., Liu, R., Xue, C., Wei, N., Deng, X. W., et al. (2016b). The red light receptor phytochrome B directly enhances substrate-E3 ligase interactions to attenuate ethylene responses. Dev. Cell 39, 597-610. doi: 10.1016/j. devcel.2016.10.020

Skirycz, A., Claeys, H., De Bodt, S., Oikawa, A., Shinoda, S., Andriankaja, M., et al. (2011). Pause-and-stop: the effects of osmotic stress on cell proliferation during early leaf development in Arabidopsis and a role for ethylene signaling in cell cycle arrest. Plant Cell 2011 (23), 1876-1888. doi: 10.1105/tpc.111.084160

Sequeira-Mendes, J., Aragüez, I., Peiró, R., Mendez-Giraldez, R., Zhang, X., Jacobsen, S. E., et al. (2014). The functional topography of the Arabidopsis genome is organized in a reduced number of linear motifs of chromatin states. Plant Cell 26, 2351-2366. doi: 10.1105/tpc.114.124578

Solano, R., Stepanova, A., Chao, Q., and Ecker, J. R. (1998). Nuclear events in ethylene signaling: a transcriptional cascade mediated by ETHYLENEINSENSITIVE3 and ETHYLENE-RESPONSE-FACTOR1. Genes Dev. 12, 3703-3714. doi: 10.1101/gad.12.23.3703

Solymosi, K., and Schoefs, B. (2010). Etioplast and etio-chloroplast formation under natural conditions: the dark side of chlorophyll biosynthesis in angiosperms. Photosynth. Res. 105, 143-166. doi: 10.1007/s11120-010-9568-2

Song, S., Huang, H., Gao, H., Wang, J., Wu, D., Liu, X., et al. (2014). Interaction between MYC2 and ETHYLENE INSENSITIVE3 modulates antagonism between jasmonate and ethylene signaling in Arabidopsis. Plant Cell 26, 263279. doi: $10.1105 /$ tpc. 113.120394

Song, J., Zhu, C., Zhang, X., Wen, X., Liu, L., Peng, J., et al. (2015). Biochemical and structural insights into the mechanism of DNA recognition by Arabidopsis ETHYLENE INSENSITIVE3. PLoS One 10, e0137439. doi: 10.1371/journal.pone.0137439

Sun, T. P., and Gubler, F. (2004). Molecular mechanism of gibberellin signaling in plants. Annu. Rev. Plant Biol. 55, 197-223. doi: 10.1146/ annurev.arplant.55.031903.141753

Vandenbussche, F., Vaseva, I., Vissenberg, K., and Van Der Straeten, D. (2012). Ethylene in vegetative development: a tale with a riddle. New Phytol. 194, 895909. doi: 10.1111/j.1469-8137.2012.04100.x

Vaseva, I. I., Qudeimat, E., Potuschak, T., Du, Y., Genschik, P., Vandenbussche, F., et al. (2018). The plant hormone ethylene restricts Arabidopsis growth via the epidermis. Proc. Natl. Acad. Sci. U.S.A. 115, E4130-E4139. doi: 10.1073/ pnas. 1717649115

Wang, L., and Qiao, H. (2019). New insights in transcriptional regulation of the ethylene response in Arabidopsis. Front. Plant. Sci. 10, 790. doi: 10.3389/fpls.2019.00790 
Wasternack, C., and Song, S. (2017). Jasmonates: biosynthesis, metabolism, and signaling by proteins activating and repressing transcription. J. Exp. Bot. 68, 1303-1321. doi: 10.1093/jxb/erw443

Wawrzyńska, A., and Sirko, A. (2014). To control and to be controlled: understanding the Arabidopsis SLIM1 function in sulfur deficiency through comprehensive investigation of the EIL protein family. Front. Plant. Sci. 5, 575. doi: $10.3389 /$ fpls.2014.00575

Wawrzyńska, A., and Sirko, A. (2016). EIN3 interferes with the sulfur deficiency signaling in Arabidopsis thaliana through direct interaction with the SLIM1 transcription factor. Plant Sci. 253, 50-57. doi: 10.1016/j.plantsci.2016.09.002

Wollmann, H., Holec, S., Alden, K., Clarke, N. D., Jacques, P.É., and Berger, F. (2012). Dynamic deposition of histone variant H3.3 accompanies developmental remodeling of the Arabidopsis transcriptome. PLoS Genet. 8, e1002658. doi: 10.1371/journal.pgen.1002658

Weirauch, M. T., Yang, A., Albu, M., Cote, A. G., Montenegro-Montero, A., Drewe, P., et al. (2014). Determination and inference of eukaryotic transcription factor sequence specificity. Cell 158, 1431-1443. doi: 10.1016/j.cell.2014.08.009

Yamamuro, C., Zhu, J. K., and Yang, Z. (2016). Epigenetic modifications and plant hormone action. Mol. Plant 9, 57-70. doi: 10.1016/j.molp.2015.10.008

Yan, Z., Liu, X., Ljung, K., Li, S., Zhao, W., Yang, F., et al. (2017). Type B response regulators act as central integrators in transcriptional control of the auxin biosynthesis enzyme TAA1. Plant Physiol. 175, 1438-1454. doi: 10.1104/ pp.17.00878

Yang, Y., Ou, B., Zhang, J., Si, W., Gu, H., Qin, G., et al. (2014). The Arabidopsis mediator subunit MED16 regulates iron homeostasis by associating with EIN3/ EIL1 through subunit MED25. Plant J. 77, 838-851. doi: 10.1111/tpj.12440

Yazaki, J., Galli, M., Kim, A. Y., Nito, K., Aleman, F., Chang, K. N., et al. (2016). Mapping transcription factor interactome networks using HaloTag protein arrays. Proc. Natl. Acad. Sci. U.S.A. 113, E4238- E4247. doi: 10.1073/pnas.1603229113

Yi, K., Menand, B., Bell, E., and Dolan, L. (2010). A basic helix-loop-helix transcription factor controls cell growth and size in root hairs. Nat. Genet. 42, 264-267. doi: 10.1038/ng.529

Zemlyanskaya, E. V., Levitsky, V. G., Oshchepkov, D. Y., Grosse, I., and Mironova, V. V. (2017b). The interplay of chromatin landscape and DNA-binding context suggests distinct modes of EIN3 regulation in Arabidopsis thaliana. Front. Plant. Sci. 7, 2044. doi: 10.3389/fpls.2016.02044

Zhang, L., Li, Z., Quan, R., Li, G., Wang, R., and Huang, R. (2011). An AP2 domain-containing gene, ESE1, targeted by the ethylene signaling component EIN3 is important for the salt response in Arabidopsis. Plant Physiol. 157, 854865. doi: 10.1104/pp.111.179028

Zhang, F., Qi, B., Wang, L., Zhao, B., Rode, S., Riggan, N. D., et al. (2016). EIN2dependent regulation of acetylation of histone H3K14 and non-canonical histone H3K23 in ethylene signalling. Nat. Commun. 7, 13018. doi: 10.1038/ ncomms 13018
Zhang, F., Wang, L., Ko, E. E., Shao, K., and Qiao, H. (2018a). Histone deacetylases SRT1 and SRT2 interact with ENAP1 to mediate ethyleneinduced transcriptional repression. Plant Cell 30, 153-166. doi: 10.1105/ tpc. 17.00671

Zhang, X., Ji, Y., Xue, C., Ma, H., Xi, Y., Huang, P., et al. (2018b). Integrated regulation of apical hook development by transcriptional coupling of EIN3/ EIL1 and PIFs in Arabidopsis. Plant Cell 30, 1971-1988. doi: 10.1105/ tpc. 18.00018

Zhang, F., Wang, L., Qi, B., Zhao, B., Ko, E. E., Riggan, N. D., et al. (2017). EIN2 mediates direct regulation of histone acetylation in the ethylene response. Proc. Natl. Acad. Sci. U.S.A. 114, 10274-10279. doi: 10.1073/pnas.1707937114

Zhang, X., Zhu, Z., An, F., Hao, D., Li, P., Song, J., et al. (2014). Jasmonate-activated MYC2 represses ETHYLENE INSENSITIVE3 activity to antagonize ethylenepromoted apical hook formation in Arabidopsis. Plant Cell 26, 1105-1117. doi: 10.1105/tpc.113.122002

Zhong, S., Shi, H., Xue, C., Wang, L., Xi, Y., Li, J., et al. (2012). A molecular framework of light-controlled phytohormone action in Arabidopsis. Curr. Biol. 22, 1530-1535. doi: 10.1016/j.cub.2012.06.039

Zhong, S., Shi, H., Xue, C., Wei, N., Guo, H., and Deng, X. W. (2014). Ethyleneorchestrated circuitry coordinates a seedling's response to soil cover and etiolated growth. Proc. Natl. Acad. Sci. U.S.A. 111, 3913-3920. doi: 10.1073/ pnas. 1402491111

Zhong, S., Zhao, M., Shi, T., Shi, H., An, F., Zhao, Q., et al. (2009). EIN3/EIL1 cooperate with PIF1 to prevent photo-oxidation and to promote greening of Arabidopsis seedlings. Proc. Natl. Acad. Sci. U.S.A. 106, 21431-21436. doi: 10.1073/pnas.0907670106

Zhu, Z., An, F., Feng, Y., Li, P., Xue, L., A. M., et al. (2011). Derepression of ethylenestabilized transcription factors (EIN3/EIL1) mediates jasmonate and ethylene signaling synergy in Arabidopsis. Proc. Natl. Acad. Sci. U.S.A. 108, 12539-12544. doi: 10.1073 /pnas.1103959108

Zhu, Z., and Lee, B. (2015). Friends or foes: new insights in jasmonate and ethylene co-actions. Plant Cell Physiol. 56, 414-420. doi: 10.1093/pcp/pcu171

Conflict of Interest Statement: The authors declare that the research was conducted in the absence of any commercial or financial relationships that could be construed as a potential conflict of interest.

Copyright (c) 2019 Dolgikh, Pukhovaya and Zemlyanskaya. This is an open-access article distributed under the terms of the Creative Commons Attribution License (CC BY). The use, distribution or reproduction in other forums is permitted, provided the original author(s) and the copyright owner(s) are credited and that the original publication in this journal is cited, in accordance with accepted academic practice. No use, distribution or reproduction is permitted which does not comply with these terms. 